\title{
Psychological wellbeing in survivors of cardiac arrest, and its relationship to neurocognitive function
}

Ms Siân E. Davies ${ }^{1}$, MSc, PhD Student.

Ms Megan Rhys², BSc, Research Paramedic.

Dr Sarah E. Voss ${ }^{3}$, PhD, Senior Research Fellow in Emergency Care.

Ms Rosemary Greenwood ${ }^{4}$, MSc, Senior Statistician.

Dr Matthew Thomas ${ }^{1}, \mathrm{MB}$ ChB, Consultant in Anaesthesia and Critical Care.

Prof Jonathan R. Benger ${ }^{3,5}$, MD, Professor of Emergency Care and Consultant in Emergency Medicine.

${ }^{1}$ Behavioural and Clinical Neuroscience Institute, Univeristy of Cambridge

${ }^{2}$ South Western Ambulance Service NHS Foundation Trust.

${ }^{3}$ Faculty of Health and Applied Sciences, University of the West of England, Bristol. ${ }^{4}$ Research Design Service - South West, Education Centre, University Hospitals Bristol NHS Foundation Trust.

${ }^{5}$ University Hospitals Bristol NHS Foundation Trust

Corresponding Author:

Dr Sarah Voss, Senior Research Fellow in Emergency Care, Faculty of Health and Applied Sciences, University of the West of England, Glenside Campus (1H14), Blackberry Hill, Bristol, BS16 1DD. Tel: 0117328 8906. Email: sarah.voss@uwe.ac.uk

Keywords:

Death, Sudden, Cardiac; Quality of Life; Anxiety. 


\section{Abstract}

Objective: To characterise psychological wellbeing in survivors of out-of-hospital cardiac arrest (OHCA), and examine its relationship to cognitive function.

Patients: Forty-one highly functioning cardiac arrest survivors were drawn from the follow-up cohort of a randomised controlled trial of initial airway management in OHCA (ISRCTN:18528625).

Design: Psychological wellbeing was assessed with a self-report questionnaire (the Depression Anxiety and Stress Scale; DASS) and cognitive function was examined using the Delayed Matching to Samples (DMS) test from the Cambridge Neuropsychological Test Automated Battery (CANTAB).

Results: Mean anxiety levels were significantly higher in this patient group than normative data drawn from the general population $(p=0.046)$. Multiple regression analyses showed that cognitive function, measured by the DMS, did not predict any of the DASS scales.

Conclusions: Anxiety plays an important role in determining perceived QoL in high functioning survivors, but psychological wellbeing is unrelated to cognitive function in this group. To achieve a comprehensive assessment of wellbeing, resuscitation research should consider outcomes beyond neurological function alone. 


\section{Introduction}

Overall survival rates from out-of-hospital cardiac arrest (OHCA) are low, but those who do recover are often judged to have a favourable outcome. ${ }^{1}$ The International Liaison Committee on Resuscitation recommends that epidemiological data define the neurological function of OHCA survivors using the Cerebral Performance Category (CPC) scale. ${ }^{2}$ However the CPC scale is a very coarse measure of function; it lacks the sensitivity needed to detect subtle differences in neurological impairment among survivors. ${ }^{3}$

Hypoxic brain injury is a common consequence of cardiac arrest, due to the brain's vulnerability to an interrupted circulation. ${ }^{4}$ Memory is the most commonly reported disturbance in OHCA survivors, ${ }^{5}$ which has been related to the particular sensitivity of the hippocampus and medial temporal lobe to hypoxia. ${ }^{6}$

Studies have found significant relationships between complaints of cognitive dysfunction and self-reported health-related quality-of-life (HRQoL) ${ }^{7}$. However, a good survival outcome should not be defined by the presence or absence of impairment, but by "a state of complete physical, mental and social well-being". 8 Resuscitation research is beginning to adopt this multifaceted definition of survival outcomes with HRQoL assessments in OHCA. ${ }^{9}$ However, current measures are weighted towards physical functioning, ${ }^{10}$ and often fail to evaluate emotional distress. Indeed, Elliott and colleagues state that despite what was reportedly a good HRQoL, "a substantial burden of morbidity exists in the survivor population". 9

The psychological aftermath of OHCA has not been adequately researched. A recent review stated that research is hindered by a lack of methodological consensus, yet the prevalence of depression and anxiety in cardiac arrest survivors could be as high as $45 \%$ and $61 \%$ respectively. ${ }^{11}$ The use of non-specific clinical scales that employ normative cut-offs, ${ }^{11}$ and a failure to evaluate the impact of stress on survivors, ${ }^{12}$ have limited the potential to comprehensively characterise post-arrest wellbeing. The Depression, Anxiety and Stress Scale addresses these problems, ${ }^{13}$ and was therefore used in this research.

The aim of this study was to characterise psychological wellbeing in high-functioning survivors of out-of-hospital cardiac arrest (OHCA), and examine its relationship to cognitive function. Our primary hypothesis was that OHCA survivors would report poorer psychological wellbeing than normal populations. As previous research has found that post-arrest cognitive deficits impact perceived quality-of-life (QoL), ${ }^{7}$ our secondary hypothesis was that cognitive function, measured by memory performance, would predict wellbeing in cardiac arrest survivors. 


\section{Methods}

Patients were drawn from the 3-month follow-up assessment of REVIVE-Airways; a randomised trial of alternative initial airway management strategies in adult nontraumatic out of hospital cardiac arrest (OHCA). ${ }^{14}$ Approval was obtained from the Cambridge Central NHS Research Ethics Committee.

Adult (18 years and older) OHCA survivors, with capacity to give informed consent at discharge from hospital, were included in the REVIVE-Airways follow-up assessment. Forty-nine patients were eligible for inclusion. Seven declined participation, and one patient with a pre-existing neurological deficit was excluded from the study.

Patients were assessed using the Depression, Anxiety and Stress Scale (DASS), ${ }^{13}$ and the Delayed Matching to Samples (DMS) test from The Cambridge neuropsychological test automated battery (CANTAB) assessment. ${ }^{15}$ They also completed the Mini Mental State Examination (MMSE). ${ }^{16}$ Further details of these are given in the Supplemental Appendix 1.

Data collection took place over one year, and all patients were assessed during a one-hour home visit conducted by the same investigator (SED) who was blinded to allocation in the REVIVE Airways study

\section{Data Analysis}

All analyses were completed using the SPSS Statistics package, Version 20.0. DASS normative data comparisons were those reported by Crawford and Henry. ${ }^{17}$

All DASS scales were found to be positively skewed, replicating previous findings. ${ }^{17}$ Skewed variables were subjected to a square root transformation to improve normality, linearity and homoscedasticity of residuals for regression analyses. ${ }^{18}$

Seven patients were excluded from the DMS analyses, and one from all DASS analyses due to insufficient data to allow accurate estimations on each scale. ${ }^{13}$ 


\section{Results}

The characteristics of the final sample of 41 patients are shown in Table 1 . The majority of patients were assessed at 3 months post arrest (87.8\%); four were assessed at 4 months, and one at 9 months. All patients were highly functioning, defined as Cerebral Performance Category 1 at follow-up. The mean Mini Mental State Examination (MMSE) score was 27.5 ( $S D=2.37 ; n=40)$.

Table 2 presents the means, medians, standard deviations and ranges for the DASS scores of all OHCA survivors. The means for OHCA survivors and normative data on each DASS scale are shown in Figure 1. OHCA survivors tended to report higher levels of depression and anxiety, and lower levels of stress, than that of the normal population. However, independent samples t-tests showed that only the observed difference in anxiety between the OHCA survivors and normative data was significant $(p=.046)$.

Hierarchical multiple regression analyses were performed on the entire patient population, controlling for the effect of age. Age was entered into the first step and all DMS variables were entered into the second step. Neither age nor any of the DMS variables, were found to significantly predict any DASS scales (all cases $p>0.05$ ). 


\section{Discussion}

We have shown that three months after an out-of-hospital cardiac arrest highfunctioning survivors do not report levels of depression or stress that are significantly different to the normal population. These findings extend the HRQoL literature with more detailed psychological assessments, and generally support their assertion that OHCA survivors experience a relatively good quality of life postOHCA. ${ }^{9,19}$

However, the study observed an elevated mean anxiety score for OHCA survivors. This is in keeping with the review by Wilder Schaaf and colleagues, ${ }^{11}$ and qualitative research, which demonstrate a particularly high prevalence of anxiety compared with depression. ${ }^{20}$ Indeed, informal feedback from patients suggested that the difficulty of recovering emotionally from their experience far exceeded any physical challenge of rehabilitation. A higher mean level of anxiety despite a normal level of depression and stress suggests that using non-specific measures could omit individuals in need of psychological support. This highlights the need for clinicians to be aware of the limitations of current assessment tools.

In our study memory performance failed to predict psychological outcomes, which conflicts with some of the previously published research. ${ }^{7}$ We were unable to demonstrate a convincing relationship between cognitive function and psychological wellbeing in this cohort, which may be attributable to their high-functioning status. Indeed, other authors have also been unable to demonstrate an association with QoL in survivors of cardiac arrest. ${ }^{21,22}$ It is likely that for highly functioning individuals, other factors are more influential in QoL perceptions. Therefore, in practice, documenting neurological parameters alone may not be sufficient to assess patient wellbeing; reporting outcomes beyond cognitive function should be considered. ${ }^{23}$

\section{Limitations and considerations}

REVIVE-Airways was a feasibility study, ${ }^{14}$ and consequently the relatively small sample size is a significant limitation. A larger trial would be valuable to explore this area further. Normative data used for comparison were derived from a general adult population with a mean age of 40 years, which is somewhat different to our study group. However, the influence of gender, occupation, education and age on DASS scores is known to be very low, which is reassuring. ${ }^{17}$

In addition, the stipulations of the approving NHS ethics allowed only the inclusion of patients with capacity, and therefore we were unable to study other survivors, though there were very few of these. Other authors have highlighted that QoL research on patients who recover poorly from OHCA is scarce..$^{9}$ It is crucial that these patients become adequately represented in the literature, particularly when considering the relationship between psychological wellbeing and neurocognitive function.

It is also important to consider alternative explanations for our findings. Firstly, a 
number of patients were on anti-depressant medication, which could influence selfreported mood. Moreover, it is paramount to remember the significance of having survived when considering the psychological impact of OHCA. Positive attitudes to survival could counter the distresses of other patients in group-level analyses, and account for the similarities in mood. In this case, qualitative research would be of considerable value. Exploring strategies that enable a positive outlook may be useful in helping patients who require additional post-arrest psychological support. ${ }^{24}$ 


\section{Conclusions}

Levels of stress are higher than levels of anxiety for OHCA survivors, but not higher that for the normal population. However, anxiety is significantly elevated in high functioning survivors of OHCA .In addition, psychological wellbeing appears unrelated to any resultant cognitive deficit in this patient cohort. Larger studies are necessary to confirm these findings, however for a comprehensive assessment of wellbeing resuscitation research should consider aspects of outcome beyond neurological function. There is potential for the development of an OHCA-specific QoL assessment tool.

\section{Acknowledgements}

We are grateful to all the patients who participated in the REVIVE-Airways 3-month follow-up assessment, and to South Western Ambulance Service NHS Foundation Trust for its support of this study.

\section{Conflicts of interest}

The authors all declare no conflict of interest.

\section{Ethical Adherence}

National Research Ethics Service (NRES) approval for this trial was obtained from Cambridge Central Research Ethics Committee (11-EE-0407), and the trial is registered on the International Standard Randomised Controlled Trial Registry (ISRCTN: 18528625).

\section{Role of the funding source}

This article presents independent research funded by the National Institute for Health Research (NIHR) under its Research for Patient Benefit (RfPB) Programme (Grant Reference Number PB-PG-0110-20288). The views expressed are those of the author(s) and not necessarily those of the NHS, the NIHR or the Department of Health. The funding body did not influence the design, conduct, analysis or reporting of this study. 


\section{References}

1. Pell JP, Corstorphine M, McConnachie A, Waker NL, Caldwell JC, Marsden AK, Grubb NR, Cobbe SM. Post-discharge survival following pre-hospital cardiopulmonary arrest due to cardiac aetiology: temporal trends and impact of changes in clinical management. Eur Heart J 2006;27:406-412.

2. Cummins RO, Chamberlain D, Hazinski MF, Nadkarni V, Kloeck W, Kramer E, Becker L, Robertson C, Koster R, Zaritsky A, Bossaert L, Ornato JP, Callanan V, Allen M, Steen P, Connolly B, Sanders A, Idris A, Cobbe S. Recommended guidelines for reviewing, reporting, and conducting research on in-hospital resuscitation: the in-hospital 'Utstein Style'. Ann Emerg Med 1997;29:650-679.

3. Nolan JP, Neumar RW, Adrie C, Aibiki M, Berg RA, Böttiger BW, Callaway C, Clark RSB, Geocadin RG, Jauch EC, Kern KB, Laurent I, Longstreth WT, Merchant RM, Morley P, Morrison $L$, Nadkarni V, Peberdy MA, Rivers EP, Rodriguez-Nunez A, Sellke FW, Spaulding C, Sunde K, Hoek TV. Post-cardiac arrest syndrome: Epidemiology, pathophysiology, treatment, and prognostication. A Scientific Statement from the International Liaison Committee on Resuscitation; the American Heart Association Emergency Cardiovascular Care Committee; the Council on Cardiovascular Surgery and Anesthesia; the Council on Cardiopulmonary, Perioperative, and Critical Care; the Council on Clinical Cardiology; the Council on Stroke. Resuscitation 2008;79:350-379.

4. Cervós-Navarro J, Diemer NH. Selective vulnerability in brain hypoxia. Crit Rev Neurobiol 1991; 6:149-182.

5. Sauve MJ, Walker JA, Massa SM, Winkle RA, Scheinman MM. Patterns of cognitive recovery in sudden cardiac arrest survivors: the pilot study. Heart and Lung: The Journal of Acute and Critical Care 1996;25:172-181.

6. Petito CK, Feldmann E, Pulsinelli WA, Plum F. (1987). Delayed hippocampal damage in humans following cardiorespiratory arrest. Neurology 1987;37:12811286.

7. Middelkamp W, Moulaert VRMP, Verbunt JA, van Heugten CM, Bakx WG, Wade DT. Life after survival: Long-term daily life functioning and quality of life of patients with hypoxic brain injury as a result of a cardiac arrest. Clin Rehabil 2007;21:425-431.

8. World Health Organisation. Constitution of World Health Organisation. Geneva: WHO. 1948.

9. Elliott VJ, Rodgers DL, Brett SJ. Systematic review of quality of life and other patient-centred outcomes after cardiac arrest survival. Resuscitation 2011;82:247-256.

10. Ware JE, Sherbourne CD. The MOS 36-item short-form health survey (SF-36): I. Conceptual framework and item selection. Med Care 1992;30:473- 483. 
11. Wilder Schaaf KP, Artman LK, Peberdy MA, Walker WC, Ornato JP, Gossip MR, Kreutzer JS. (2013). Anxiety, depression, and PTSD following cardiac arrest: A systematic review of the literature. Resuscitation 2013; 84:873-877

12. Gamper G, Willeit M, Sterz F, Herkner H, Zoufaly A, Hornik K, Havel C, Laggner AN. Life after death: posttraumatic stress disorder in survivors of cardiac arrestprevalence, associated factors, and the influence of sedation and analgesia. Crit Care Med 2004;32:378-383.

13. Lovibond SH, Lovibond PF. Manual for the Depression Anxiety Stress Scales. Second Edition. Sydney: Psychology Foundation. 1995.

14. Benger JR, Voss S, Coates D, Greenwood R, Nolan J, Rawstorne S, Rhys M, Thomas M. Randomised comparison of the effectiveness of the laryngeal mask airway supreme, i-gel and current practice in the initial airway management of prehospital cardiac arrest (REVIVE-Airways): a feasibility study research protocol. BMJ Open 2013; 3:e002467. doi:10.1136/bmjopen-2012- 002467

15. Fray PJ, Robbins TW, Sahakian BJ. Neuropsychological applications of CANTAB. Int J Geriatr Psychiatry 1996;11:329-336.

16. Folstein MF, Folstein SE, McHugh PR. "Mini-mental state". A practical method for grading the cognitive state of patients for the clinician. J Psychiatr Res 1975;12:189-198.

17. Crawford JR, Henry JD. The depression, anxiety and stress scales (DASS): Normative data and latent structure in a large non-clinical sample. Br J Clin Psychol 2003;42:111-131.

18. Field A. Discovering statistics using SPSS. Second Edition. London, England: Sage. 2005.

19. Deasy C, Bray J, Smith K, Harriss L, Bernard S, Cameron P. Functional outcomes and quality of life of young adults who survive out-of-hospital cardiac arrest. Emerg Med J 2013;30:532-537.

20. Palacios-Ceña D, Losa-Iglesias ME, Salvadores-Fuentes P, Fernández-de-las-Peñas C. Sudden cardiac death: The perspectives of Spanish survivors. Nursing and Health Sciences 2011;13:149-155.

21. Torgersen J, Strand K, Bjelland TW, Klepstad P, Kvåle R, Søreide E, WentzelLarsen T, Flaatten $\mathrm{H}$. Cognitive dysfunction and health-related quality of life after a cardiac arrest and therapeutic hypothermia. Acta Anaesthesiol Scand 2010;54:721-728.

22. Hsu JWY, Madsen CD, Callaham ML. Quality-of-life and formal functional testing of survivors of out-of-hospital cardiac arrest correlates poorly with traditional neurological outcome scales. Ann Emerg Med 1996;28:597-605. 
23. Langhelle A, Nolan J, Herlitz J, Castren M, Wenzel V, Soreide E, Engdahl J, Steen PA. Recommended guidelines for reviewing, reporting, and conducting research on post-resuscitation care: the Utstein style. Resuscitation 2005;66:271-283.

24. Bremer A, Dahlberg K, Sandman L. To survive out-of-hospital cardiac arrest: A search for meaning and coherence. Qualitative Health Research 2009;19:323338. 
Table 1.

Patient Characteristics.

\begin{tabular}{|c|c|}
\hline Patients & $\mathrm{n}=\mathbf{4 1}$ \\
\hline Mean age in years (SD) & $63(12.50)$ \\
\hline Number male $(\%)$ & $31(76)$ \\
\hline \multicolumn{2}{|l|}{ Initial Rhythm } \\
\hline VF/Pulseless VT (\%) & $26(63)$ \\
\hline PEA $(\%)$ & $3(7)$ \\
\hline $\operatorname{ASY}(\%)$ & $0(0)$ \\
\hline Unknown (\%) & $12(29)$ \\
\hline Mean time to ROSC in minutes (SD) $(n=28)$ & $9(0.005)$ \\
\hline Mean length of ICU stay in days (SD) $(n=28)$ & $6(6.76)$ \\
\hline Mean length of hospital stay in days (SD) $(n=32)$ & $13(9.73)$ \\
\hline Received Therapeutic Hypothermia (\%) $(n=33)$ & $21(63.6 \%)$ \\
\hline Received Reperfusion Therapy $(\%)(n=28)$ & $23(82.1 \%)$ \\
\hline
\end{tabular}

Table 2.

Summary of descriptive statistics of self-reported DASS scores

\begin{tabular}{|c|c|c|c|c|c|c|c|c|c|c|}
\hline & & & & & & \multicolumn{5}{|c|}{ Percentage in each DASS category } \\
\hline & $\mathrm{N}$ & Median & Mean* & $\mathrm{SD}^{*}$ & Range & Normal & Mild & Moderate & Severe & $\begin{array}{c}\text { Extremely } \\
\text { Severe }\end{array}$ \\
\hline Depression & 40 & 4.5 & 6.63 & 8.94 & $0-40$ & 80 & 5 & 5 & 5 & 5 \\
\hline Anxiety & 40 & 4.0 & 5.88 & 7.19 & $0-34$ & 80 & 2.5 & 5 & 7.5 & 5 \\
\hline Stress & 40 & 5.5 & 8.28 & 8.79 & $0-34$ & 82.5 & 2.5 & 7.5 & 5 & 2.5 \\
\hline Overall & 40 & 14.0 & 20.78 & 23.92 & $0-108$ & & & & & \\
\hline
\end{tabular}

${ }^{\star}$ Crawford and Henry (2003) reported normative mean (SD) depression score= 5.55 (7.48);

anxiety=3.56 (5.39); stress=9.27 (8.04); overall=18.38 (18.82). 


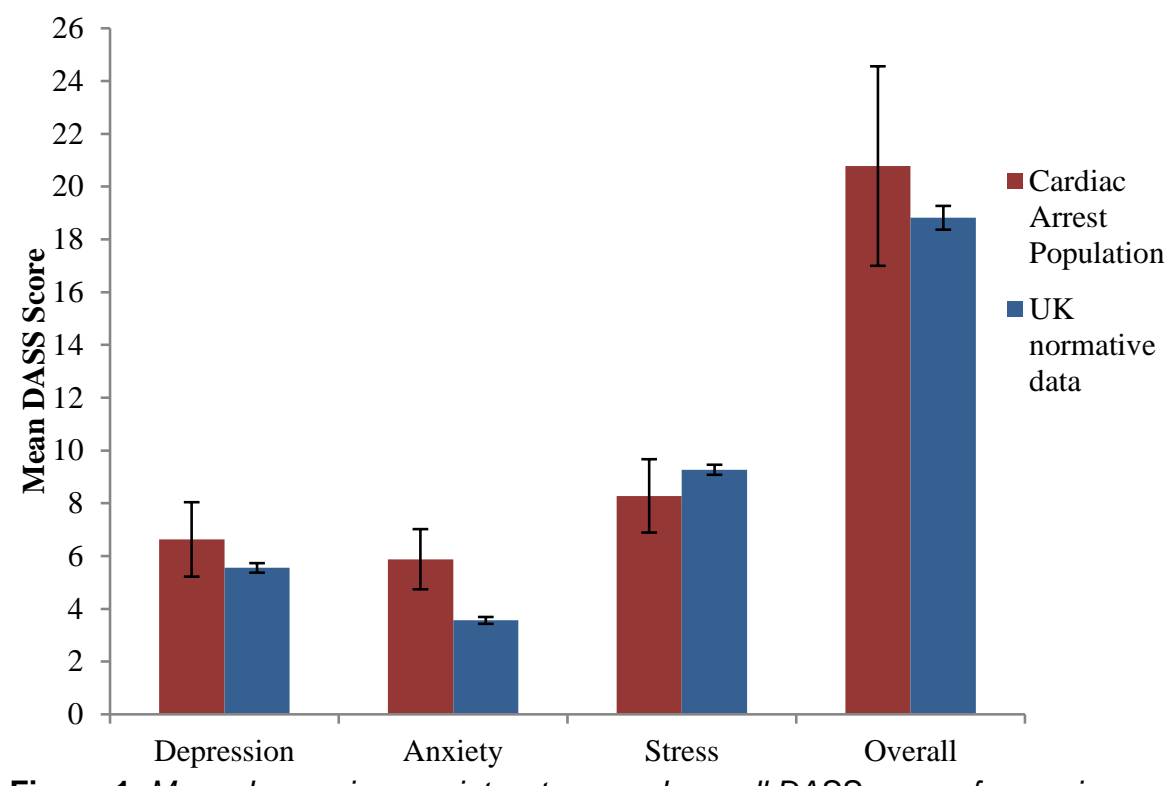

Figure 1: Mean depression, anxiety, stress and overall DASS scores for survivors compared to United Kingdom (UK) normative data. Error bars denote one standard error. 